livraisons

d'Histoire

de l'Architecture

\section{Livraisons de l'histoire de l'architecture}

$38 \mid 2019$

Varia III

\title{
Sur les traces de la première cathédrale d'Alger
}

On the way to the first cathedral of Alger

Auf den Spuren der ersten Kathedrale von Algier

\section{Amel Bellala}

\section{OpenEdition}

\section{Journals}

Édition électronique

URL : http://journals.openedition.org//ha/1488

DOI : $10.4000 /$ lha.1488

ISSN : 1960-5994

\section{Éditeur}

Association Livraisons d'histoire de l'architecture - LHA

Édition imprimée

Date de publication : 15 décembre 2019

Pagination : 63-73

ISSN : $1627-4970$

\section{Référence électronique}

Amel Bellala, «Sur les traces de la première cathédrale d'Alger », Livraisons de l'histoire de l'architecture [En ligne], 38 | 2019, mis en ligne le 02 janvier 2021, consulté le 06 février 2021. URL : http:// journals.openedition.org//ha/1488; DOl : https://doi.org/10.4000/lha.1488 


\section{SUR LES TRACES DE LA PREMIÈRE CATHÉDRALE D’ALGER}

\section{Le contexte historique et urbain}

Alger en 1830 est inadaptée à la façon de vivre des Français et à leurs besoins. Durant les dix premières années, la question de l'occupation définitive reste en discussion ${ }^{1}$. De ce fait, les Français ne détruisent que ce qui doit nécessairement disparaître et n'édifient que des constructions provisoires administratives et militaires. La déclivité importante du périmètre de l'ancienne ville ${ }^{2}$ ne permet que l'utilisation de la basse ville qui longe la mer. Toute la partie supérieure, résidentielle, reste longtemps presque intacte. Les premières destructions sont justifiées par le fait que les militaires désirent disposer d'un espace de rassemblement et sans doute d'un point de contrôle central pour surveiller l'ensemble de la ville. La place baptisée "Louis-Philippe " ne peut être établie sans des démolitions inévitables au cour de la ville, lieu le plus adéquat pour affirmer la présence et la puissance française à Alger, d'autant plus que les forces militaires sont installées dans les palais limitrophes notamment le palais de Djenina, ancien centre du pouvoir. Sur le plan cultuel, les seize aumôniers militaires qui accompagnent les troupes trouvent peu de catholiques à Alger. À cette époque, trois édifices sont réservés à la pratique de ce culte : l'ancienne chapelle du bagne des lions à Bab azoun, où ils s'installent en premier, la chapelle du consulat de France et la chapelle du consulat d'Espagne. L'affectation du palais Dar Aziza, situé au quartier de Ketchawa, à l'évêché est une première forme d'affirmation religieuse. Par ailleurs, en 1832, trois mosquées subissent une conversion fonctionnelle: la mosquée Ketchawa devenue la cathédrale d'Alger Saint-Philippe, la mosquée Ali Betchnin devenue Notre-Dame de la Victoire et la mosquée El Berrani qui prend le nom d'église Sainte-Anne. Afin d'affirmer cette décision, et dans la continuité des travaux de réaménagement, le quartier de Ketchawa connaît une mutation urbaine importante, marquée par la démolition du passage couvert Sabbat Eddiouen et des constructions reliant la place d'armes à l'évêché d'une part, mais aussi celle de l'ensemble des bâtiments situés entre l'évêché et la mosquée Ketchawa, devenue cathédrale, donnant ainsi naissance à la place de l'évêché (ill. 1).

1. Léon Blondel, Nouvel aperçu sur l'Algérie: Trois nécessités an Afrique : conserver, pacifier, coloniser, éditions Bohaire, Paris, 1838, p. 101-102.

2. Depuis le port jusqu’à la citadelle, la ville se développe le long des $650 \mathrm{~m}$ sur une différence de niveau qui est de $117 \mathrm{~m}$. 


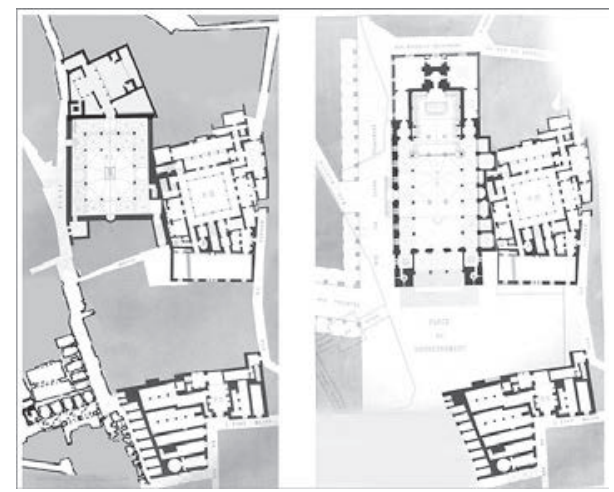

Ill. 1 : « Restitution urbaine du plateau Ketchawa. À gauche, l'état initial conforme à l'époque ottomane, à droite, la cathédrale et la place de l'Évêché avec la projection des voiries", planche établie par Amel Bellala, Essai de restitution de la mosquée Ketchawa 1794-1840, thèse de magistère, LVAP, epau, 2018. (C) D.R.

\section{Le bâtiment initial: une mosquée ottomane}

L'histoire de la première cathédrale d'Alger est fortement liée à celle de la ville. À ses origines, on trouve la mosquée Ketchawa, qui d'après un ancien acte du Beylik $^{3}$ date du XIII ${ }^{\mathrm{e}}$ siècle. Cette première mosquée à propos de laquelle on dispose de très peu d'informations est démolie puis reconstruite vers la fin du XVIII siècle, par Hassan pacha qui l'a considérablement agrandie. C'est une opération récurrente à la fin du XVII ${ }^{\mathrm{e}}$ et durant le XVIII ${ }^{\mathrm{e}}$ siècle, où la ville d'Alger s'édifie sur elle-même du fait de la saturation urbaine ${ }^{4}$, imposant le réinvestissement des parcelles. La nouvelle mosquée est reconstruite sur une parcelle qui abritait vingt-quatre boutiques, quatre petites maisons, une taverne, un café, démolis pour libérer une parcelle importante pouvant l'accueillir. Les travaux de reconstruction sont achevés en 1794-1795 comme l'indique l'inscription sur la porte de l'édifice. Les plans à notre disposition montrent que la mosquée de Ketchawa était bordée par le hammam Mohamed Pacha à l'ouest, le plais de Hassen Pacha au nord, la rue du Divan vers le sud, des boutiques, des latrines avec chambres à l'étage et un fondouk vers l'est. Ces derniers étaient des biens Waqf au profit de la mosquée Ketchawa (ill. 2).

3. Le plus ancien manuscrit arabe datant du XIV e siècle, attribué à Husin Ban Rajab, fils du Mufti hanafite de la grande mosquée d'Alger, mentionne la présence d'une petite mosquée sur l'emplacement correspondant au plateau de Ketchawa, dont la fondation est antérieure à 1364-1365.

4. Tal Shuval, La Ville d'Alger vers la fin du XVIII e siècle. Population et cadre urbain, Paris, CNRS Éditions, 1998, p. 193-194. 


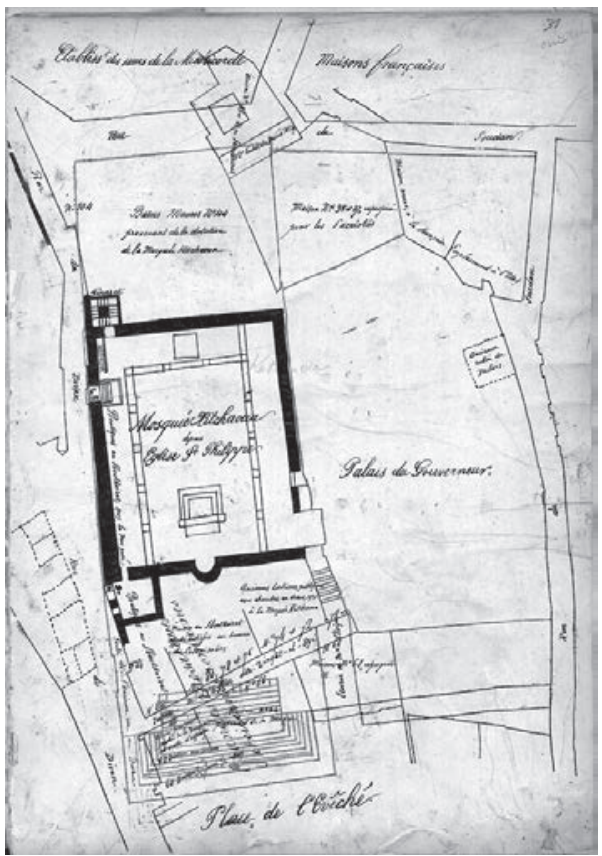

Ill. 2 : «Restitution du parcellaire du plateau Ketchawa avec projection de l'assiette de la nouvelle cathédrale ", Albert Devoulx, archives de la bibliothèque nationale d'Alger, service des manuscrits, MS.2313. (C) D.R.

\section{L'Organisation spatiale}

La salle de prière s'organise autour d'un espace central d'environ 11,50 m de côté, surmontée d'une coupole octogonale posée sur quatre trompes en coquilles. Cet espace est bordé par une galerie double, sur le côté opposé de la niche du mihrab, et fermé sur les trois côtés par des galeries immenses de $65 \mathrm{~m}$ de longueur sur 3,30 $\mathrm{m}$ de largeur, dans lesquelles sont aménagées des tribunes à mi-hauteur du sol, réservées aux femmes (ill. 3). Ce plan en longueur a pu évoquer, à une certaine période, une ressemblance étroite avec celui des églises, construite en croix latine ${ }^{5}$. Cette hypothèse ne peut être retenue car outre la mosquée Ketchawa, d'autres mosquées ont la même organisation spatiale et cette configuration se justifie par la nécessité d'augmenter la surface réservée aux fidèles. Ce dispositif, courant à Alger bien qu'étranger à l'Afrique du nord médiévale, évoque celui des mosquées à grande coupole centrale diffusé en Turquie. Les murs intérieurs sont revêtus de carreaux de porcelaine blanche et bleue, jusqu'à la hauteur de la tribune, où des armoires couvrent l'ensemble des murs. Les portes intérieures présentent des panneaux composés de pièces rapportées, chacune de couleur différente. Des inscriptions

5. R. Dokali, "La mosquée de Kerchawa ", Algérie Actualité, semaine du 5 au 11 octobre, 1969, p. 12-13. 


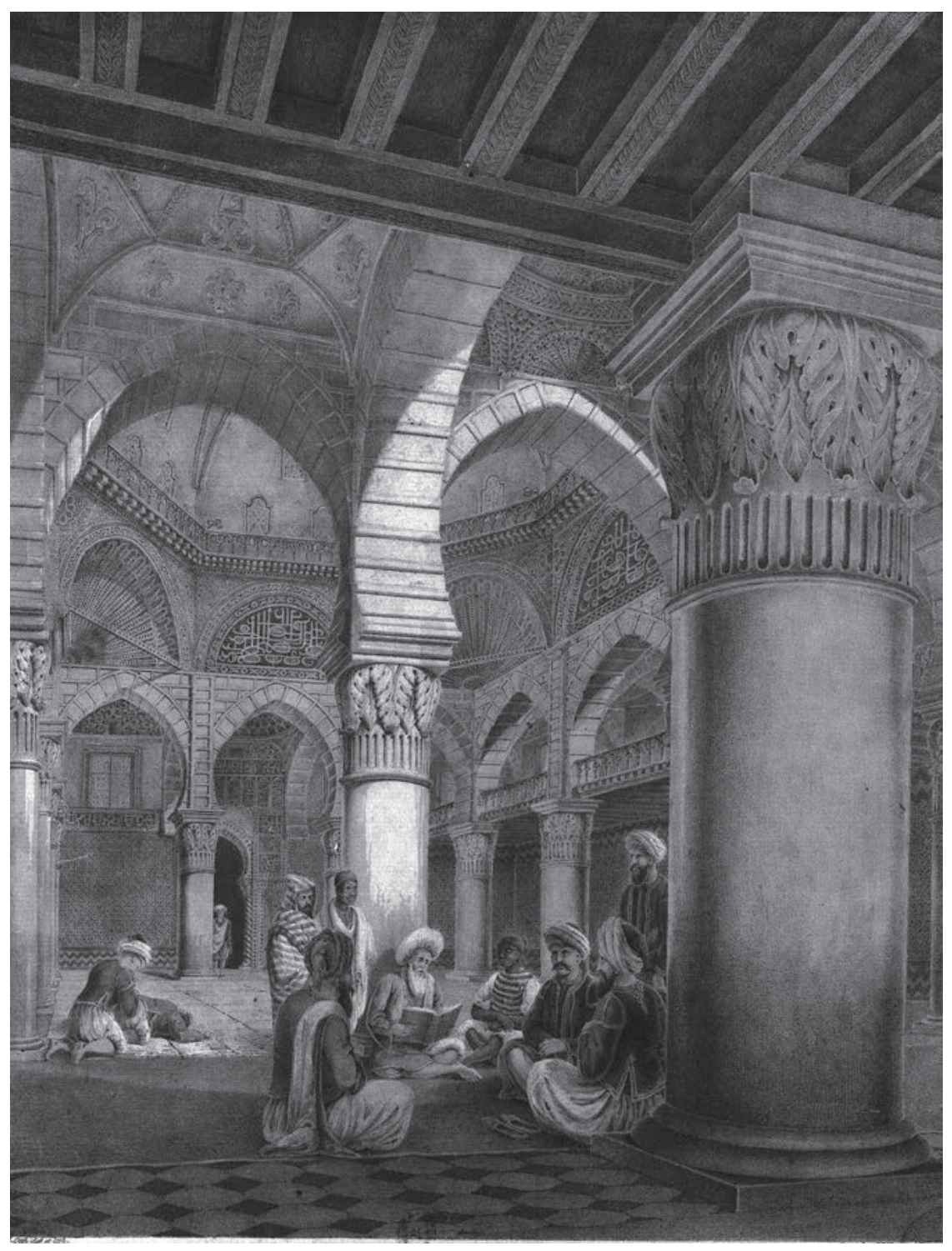

Ill. 3 : «Intérieur d'une mosquée à Alger », Adolphe Carl Otth 1838, Esquisses africaines dessinées pendant un voyage à Alger et lithographiées. Berne, J. F. Wagner, 1838-1839, planche scannée par les archives de la bibliothèque nationale d'Alger, service audiovisuel de la BNA - Document non réf. (C) D.R. 
colossales de versets du Coran, dont certaines mesurent presque un mètre de haut ${ }^{6}$, ornent les murs. Dans la vaste tribune, une place est réservée à la famille du Dey. Après la conversion du bâtiment en cathédrale, elle reviendra au gouverneur et à sa famille qui s'y rendent directement depuis le palais d'hiver par une porte de communication $^{7}$.

\section{La mosquée devient église : réadaptation spatiale et cultuelle}

La cession de la mosquée Ketchawa est la conclusion d'un double processus. Recensée ${ }^{8}$ comme appartenant au Beylik turc et gérée par les corporations religieuses, elle a été cédée par les turcs au général en chef de l'armée française en 1830, à l'instar des bâtiments appartenant au Beylik et est resté fermée pendant deux ans et demi ${ }^{9}$. Des documents conservés dans les archives de l'archevêché d'Alger ${ }^{10}$ indiquent qu'elle a servi d'entrepôt à l'armée française dès son arrivée et que son personnel a été relevé de ses fonctions dès juillet 1830 par le Dey Hussein. En 1832, le Duc de Rovigo entreprend des démarches pour sa cession. Les autorités religieuses locales désireuses de la conserver au culte musulman proposent plusieurs alternatives comme la mosquée Djamaa Djedid, dont le plan évoque celui d'une église, mais les autorités françaises rejettent ces propositions. Informés que dix mille personnes parmi la population jurent de mourir sous la coupole de Ketchawa plutôt que de consentir à sa prise ${ }^{11}$, les militaires doublent les postes de garde et confisquent les clés de l'édifice. Le 17 décembre 1832, l'occupation de la mosquée est ordonnée. La situation s'aggrave quelque peu lors de la prise en possession par l'armée française, des coups de feu sont échangés, des fidèles musulmans sont tués ou blessés à l'intérieur où ils s'étaient retranchés. L'occupation du lieu de culte par une compagnie d'infanterie scelle la prise de possession de la mosquée Ketchawa ${ }^{12}$ et malgré l'émoi de la population, les Muftis consentent à sa cession exigée dès

6. Bargès (abbé), " Notice sur la cathédrale d'Alger en 1839 ", Journal asiatique, février, troisième série, tome XI, l'imprimerie royale, Paris, 1841, p. 181-188.

7. Henri Klein, «La cathédrale d'Alger monument historique ", Algérie Catholique, $3^{\mathrm{e}}$ année, $\mathrm{n}^{\circ} 4$, Pâques 1939, p. 10-12.

8. Le capitaine du corps royal du Génie était chargé dès 1832 d'établir les éléments de constitution d'un cadastre à l'usage d'une future administration des Domaines, voir : capitaine Charles Mangay, " Note sur la propriété à Alger avant l'occupation française ", dans E. Pelissier de Raynaud, Anselin et Gautier, Annales Algériennes, 1839.

9. Archives de l'archevêché d'Alger (AAA) / dossier 283, R. Rostagny, « Petit retour au temps où notre cathédrale était une mosquée où l'on célébrait la messe de minuit à la caserne des lions ", extrait de Dernière heure, Noël 1939, p. 25.

10. Pour rappel, une grande partie des documents d'archives relatifs à l'histoire de ce monument dans ses différentes époques est conservée aux archives de l'archevêché d'Alger, casier : cathédrale SaintPhilippe, dossiers AAA/283, AAA/12, AAA/234, AAA/232.

11. Ibid.

12. Ibid. 
le 23 mai $1832^{13}$. La mosquée devenue église subit, dans un premier temps, des modifications légères pour l'adapter aux pratiques religieuses chrétiennes. La cérémonie d'inauguration a lieu lors de la messe de minuit ${ }^{14}$ le 24 décembre 1832, date de l'affectation officielle de la mosquée au culte catholique par l'abbé Colin, préfet apostolique.

\section{L'adaptation}

L'adaptation du monument aux pratiques religieuses chrétiennes se fait en deux phases. En premier lieu, des aménagements sont effectués à l'intérieur de l'ancienne mosquée par l'installation d'un nouveau mobilier et le remploi de l'ancien. La vasque des ablutions qui se trouve à l'entrée, du côté de la rue du Divan, est transformée en fonts baptismaux (ill. 5). Elle est décorée de sculptures où domine l'image du croissant. Le clocheton et la rampe en marbre du minbar sont utilisés dans la construction de la chaire à prêcher, le tabernacle est exposé sur la table qui supportait le siège de marbre de l'imam orné de colonnes torses incrustées d'onyx. On y ajoute des gestes symboliques comme l'installation d'une statue de la Vierge au mihrab qui indiquait auparavant la direction de la qibla. Dans un second temps, la principale modification réside dans le changement du sens de la prière. Un autel est aménagé dans le sens opposé au mur de la qibla en remployant la table en marbre sur laquelle se posait le siège de l'imam (ill. 4). Sur l'ordre du maréchal Bugeaud, l'église est surmontée d'une croix, comme l'indique une gravure datant de $1840-1845^{15}$ sur laquelle on distingue un percement au niveau du mihrab, qui devient la porte d'accès à la cathédrale depuis la place de l'évêché. Cet édifice illustre parfaitement la double appartenance des mosquées converties durant cette période en églises. "Ces monuments qui ont bravé les siècles ; qu'il suffit de parcourir pour en retrouver l'origine, et qui furent eux-mêmes leur propre chronologie » ${ }^{16}$. Ces églises hybrides par la pratique du culte catholique associé à une architecture et des décorations musulmanes ne semblaient pas choquer les fidèles chrétiens alors que les versets coraniques gravés en 1795 par le maître Ibrahim Djarkeli ${ }^{17}$ restent visibles en grands caractères dans la décoration intérieure du monument, sur toutes les parois, au même titre que les colonnes décorées de motifs végétaux. À l'occasion des fêtes de Pâques de 1833, la reine Marie-Amélie offre à la nouvelle église des vases sacrés et les premiers ornements sacerdotaux et le pape Grégoire XVI lui fait don d'un tableau de Carrache, représentant l'Assomption, et d'un calice d'or entouré de pierreries et décoré du coq, symbole de la vigilance ${ }^{18}$.

13. Henri Klein, "La cathédrale. Chronique du vieil Alger ", La Dépêche algérienne, 16 août 1928, p. 12.

14. Henri Klein, "La cathédrale d’Alger, monument historique ", op. cit., p. 10-12.

15. Ibid.

16. Pierre Genty de Bussy, De l'établissement des Français dans la régence d'Alger, Librairie Firmin Didot frères, Paris, 1839 , p. 49-53 et 316-320.

17. Klein, "La cathédrale d'Alger monument historique ", op. cit., p. 10.

18. Charles-André Julien, Histoire de l'Algérie contemporaine, vol. I, Presse universitaires de France, Paris, 1964, p. 91. 


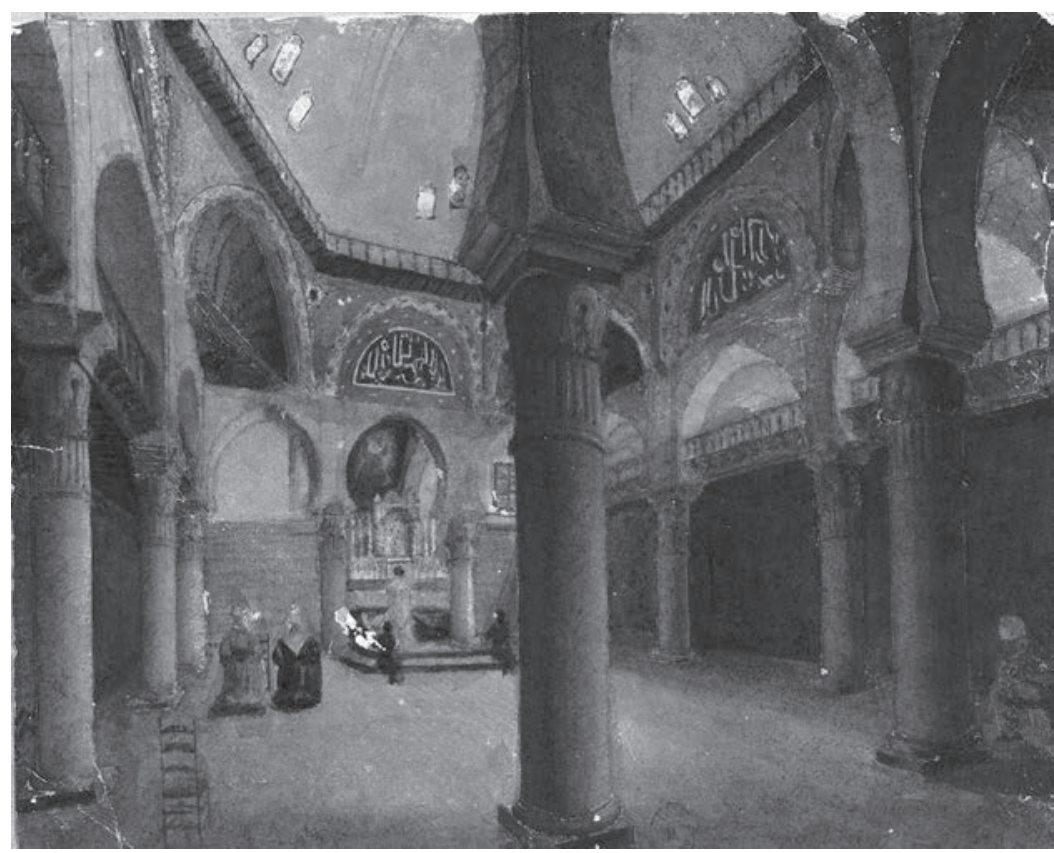

Ill. 4 : «Mosquée convertie en église », Charles Desprez, planche scannée par les archives de la bibliothèque nationale d'Alger, service audiovisuel de la BNA - Document non réf. (C) D.R.

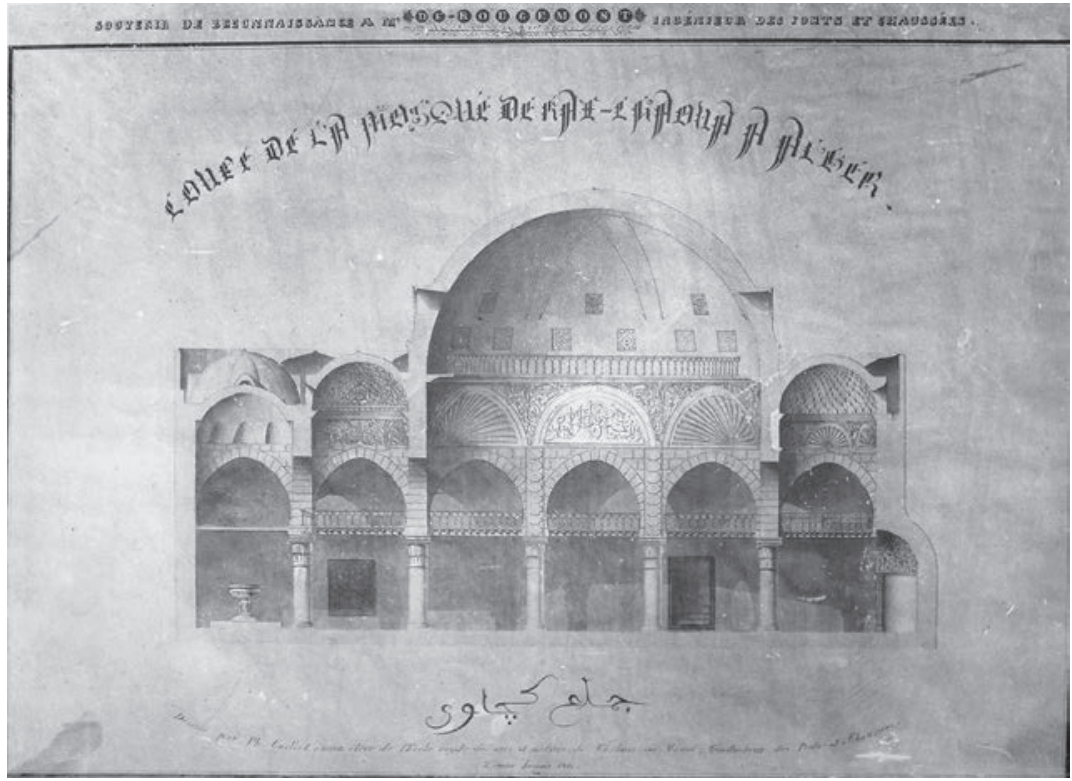

Ill. 5: "Coupe de la mosquée convertie en église », Phillipe Caillat 1839, Archives de l'archevêché d'Alger - Casier : Cathédrale Saint Philippe, Dossiers : AAA/283. ( D.R. 


\section{La démolition. S'agissait-il réellement d'une démolition totale?}

Très vite, les offices pontificaux attirent une foule importante, si bien que l'évêque, Mgr Dupuch réclame une église plus grande. Il espère d'abord pouvoir obtenir la mosquée de la Pêcherie, mais cela s'avérant impossible, il se prononce pour la construction, dans un nouveau lieu, d'une nouvelle cathédrale plus spacieuse. Les autorités politiques dont il dépend sur le plan matériel en raison du Concordat se prononcent plutôt pour la transformation totale de l'édifice existant ${ }^{19}$. Cette décision n'obéit pas seulement à un motif religieux. L'urbanisme municipal devait en premier lieu profiter de la transformation de la mosquée désaffectée pour réaliser l'élargissement et la rectification de la rue du Divan ${ }^{20}$. Le projet connaît cependant des modifications successives et importantes et finit par être abandonné en $1840^{21}$. Le gouvernement décide finalement de démolir la mosquée pour édifier sur le même emplacement une cathédrale plus grande. Des projets de transformation de l'édifice ont déjà été initiés dès les années qui ont suivi sa conversion, notamment par l'architecte des bâtiments civils Pierre-Auguste Guiauchain ${ }^{22}$. Ces premiers projets du milieu des années $1830^{23}$, remaniaient entièrement l'édifice:

"La coupole était supprimée, les galeries est et ouest étaient supprimées et l'édifice était prolongé dans les deux sens du côté de l'évêché, un grand perron rachetait la dénivellation et la façade, du moins jusqu'à la naissance des tours, était prévue comme on la voit actuellement. Le chœur, plus réduit que maintenant devait être surmonté d'un clocher central».

Ce projet a été repris en 1839 par l'architecte Amable Ravoisié dans le cadre de "L'Exploration scientifique de l'Algérie ", mais les planches qu'il a laissées étaient surtout destinées à la publication ${ }^{24}$. En 1846, Mgr Dupuch, se plaint de la suppression des tribunes et proteste également contre le fait que les plans ne lui ont jamais été présentés. Il écrit :

"Il y a plusieurs années qu'on a commencé et entrepris des réparations et constructions qui ont été mal conçues et mal conduites, de telle façon qu'on a fini par démolir l'édifice à peu près tout entier, par défaire même ce qu'on avait d'abord reconstruit ${ }^{25}$.

19. Archives de l'archevêché d'Alger, AAA/283, La Cathédrale d'Alger n'est pas une ancienne moquée, voir aussi abbé Bargès, op. cit., p. 181-88.

20. AAA/283, op. cit.

21. Ibid.

22. Nadia Oulebsir, Les usages du patrimoine. Monuments, musées et politique coloniale en Algérie (18301930), éditions de la Maison des sciences de l'homme, Paris, 2004, p. 88.

23. Ibid.

24. Amable Ravoisié, Beaux-Arts, architecture et sculpture, Exploration scientifique de l'Algérie pendant les années 1840,1841,1842, publiée par ordre du gouvernement et avec le concours d'une commission académique, Paris, Firmin-Didot, vol. 3, 1846-1851, $3^{\circ} \mathrm{V}$, PL 13, 14, 15.

25. AAA/283, op cit. 
En fait, le budget modeste réservé aux transformations imposait à l'architecte la conservation de la mosquée et son extension à l'est et à l'ouest. Le chantier de restauration de l'actuel édifice nous donne des informations précieuses. Il montre qu'une partie du mur attenant au palais de Hassen Pacha est ottomane et que l'appareillage des autres murs date de la deuxième moitié du XIX ${ }^{\mathrm{e}}$ siècle. Le système de couverture est entièrement français, contrairement à ce que prétendaient certaines études $^{26}$. La coupole ainsi que les galeries est et ouest sont supprimées et l'édifice est prolongé dans les deux sens. Un grand perron rachète la dénivellation sur la façade, du moins jusqu'à la naissance des tours, comme on la voit actuellement. Le chœur, plus réduit que l'actuel devait être surmonté d'un clocher central.

\section{La Cathédrale Saint-Philippe}

Les travaux de construction de la nouvelle cathédrale durent de 1845 à 1860 et le traitement de la façade n'est finalisé qu'à la fin du siècle. Pendant la durée des travaux, elle est remplacée momentanément par l'église Notre-Dame des Victoires. Les travaux de construction sont lancés en 1844, sur la base du projet de l'architecte du diocèse Harou-Romain. Des remaniements successifs retardent leur exécution et la pose de la première pierre de la cathédrale n'a eu lieu qu'en 1853, soit près de dix ans après la décision de destruction. Dès lors, l'architecte Jean-Baptiste Féraud est chargé du projet ${ }^{27}$. Suite à d'autres ajournements, le ministère de l'instruction publique et du culte délègue Eugène Fromageau ${ }^{28}$, architecte en chef des édifices diocésains d'Algérie pour terminer en 1856, principalement, les travaux du chœur et de l'abside. Le projet tel que le concevait cet architecte se rapprochait d'avantage du style byzantin. La cathédrale est considérablement agrandie, pour occuper environ quatre fois la surface de l'ancienne mosquée. Elle est dotée d'un escalier monumental donnant sur la place de l'évêché, pour racheter la dénivellation importante du terrain. Ces escaliers sont ornés de part et d'autres de deux tours ${ }^{29}$. Durant les travaux, le chantier a connu des problèmes liés à l'instabilité du sol sur la rue du Divan, ce qui a nécessité la mise en place de contreforts en pierre de taille sur toute la longueur du mur. À cette occasion, des sondages ont mis à jour, au-dessous des constructions ottomanes, des mosaïques de têtes d'animaux et des médaillons, appartenant probablement à des thermes romains ${ }^{30}$ et dont une partie demeure sous les fondations de la cathédrale. Les travaux de la façade se poursuivent jusqu'à la fin du XIX ${ }^{\mathrm{e}}$ siècle. Plusieurs solutions sont proposées, notamment celle d'Harou-Romain, élaborée en 1856, qui suggère l'idée de deux tours latérales, mais c'est la proposition de l'architecte Albert Ballu en 1886 qui est adoptée, avec deux tours inspirées des minarets de la mosquée El Nasser Mohammed à la citadelle du Caire et des minarets de la mosquée de Kä̈t-Bey de la période mamelouk (ill. 6).

26. Ibid.

27. AAA/283, op. cit.

28. R.P. Viard, «La cathédrale d'Alger ", Afrique du nord illustrée, no 871, 20 mai 1939, p. 22-23.

29. Ahmed Koumas et Chehrazad Nafaa, L'Algérie et son patrimoine, éditions du patrimoine, Paris, 2003, p. 132-133.

30. Nadia Oulebsir, op. cit., p. 91. 


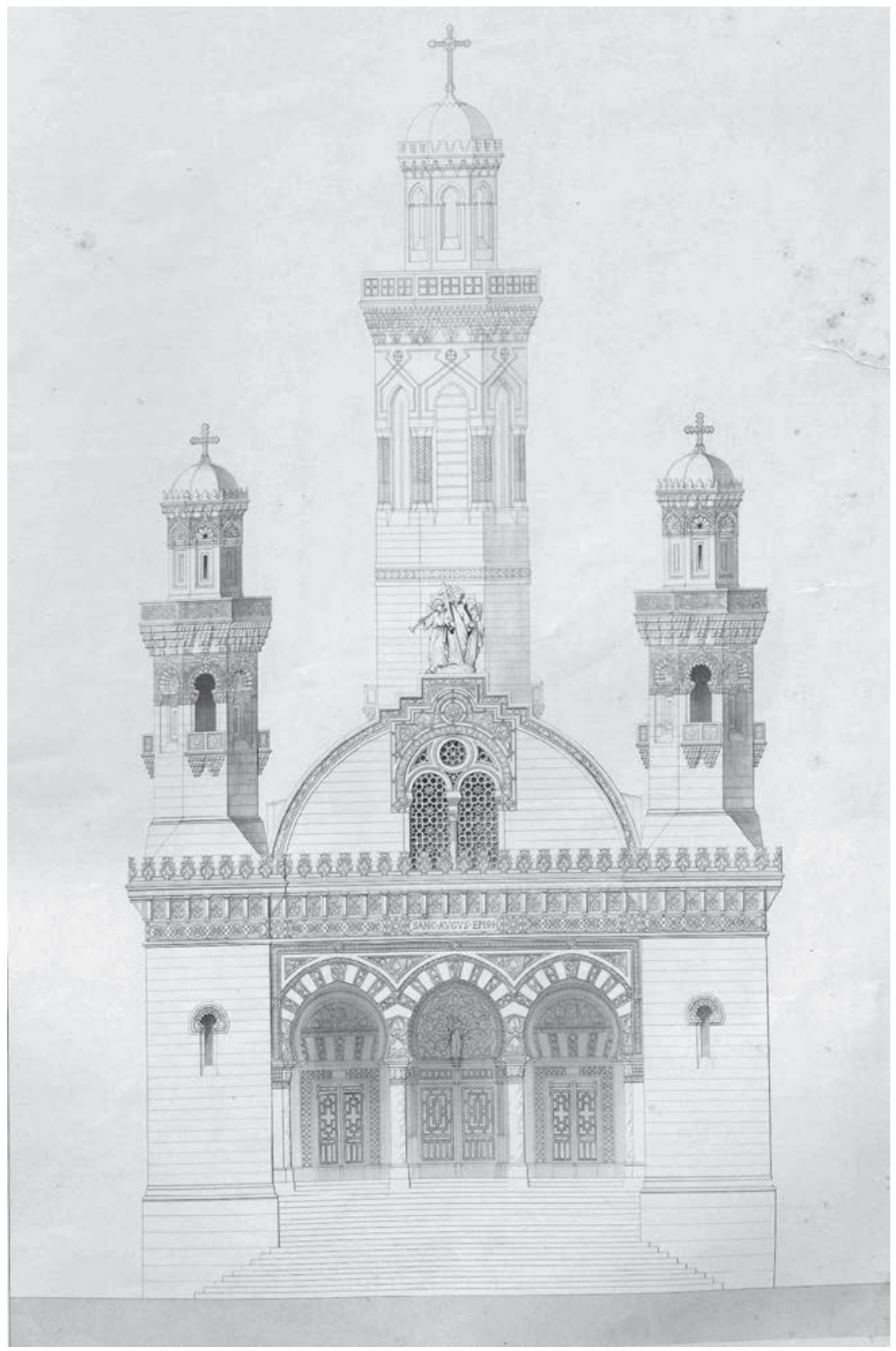

Ill. 6 : "L'Eglise Cathédrale d'Alger dessinée ", Amable Ravoisié, dans "Exploration scientifique de l'Algérie pendant les années 1840,1841, 1842 ", publiée par ordre du gouvernement et avec le concours d'une commission académique, Paris, Firmin-Didot, Volume 3, PL.18, document scanné par la MOM Lyon. (C) D.R. 
Avec le remploi des colonnes du minbar, de la vasque des ablutions de l'ancienne mosquée ainsi que des colonnes du temple de Jupiter Olympien récupérées du site antique romain de Cherchell, l'antique Césarée, et enfin la construction des tours inspirées de l'art musulman, la cathédrale prend finalement un aspect assez métissé depuis le style romano-byzantin du côté du chœur jusqu'à l'art musulman en façade principale. Les colonnes qui soutiennent les voutes de la nef centrale sont majoritairement des reproductions fidèles des originaux ${ }^{31}$. Ce mélange de différentes influences annonce l'avènement d'un nouveau mouvement stylistique aux tendances orientales en Algérie : le néo-mauresque. Très répandu au début du $\mathrm{XX}^{\mathrm{e}}$ siècle, il devient plus tard le style régional en Algérie, suite aux trois circulaires du gouverneur général Jonnart de $1904,1905,1906^{32}$ qui préconisent l'utilisation du style architectural local dans les édifices publics. Finalement, c'est seulement en 1868 que la cathédrale est consacrée ${ }^{33}$. L'église Saint-Philippe garde sa fonction de cathédrale de la ville d'Alger jusqu'à l'indépendance de l'Algérie en 1962 quand elle fut reconvertie en mosquée. Malgré le budget colossal alloué aux travaux de la transformation du bâtiment initial, le projet a été largement critiqué dans le rapport sur le projet de la nouvelle ville d'Alger en 1858. La cathédrale n'a jamais été véritablement intégrée dans la partie centrale de la ville qui manquait de grands espaces pour les réunions officielles et les grandes fêtes et est restée située au milieu d'un habitat traditionnel majoritairement musulman ${ }^{34}$. L'aspect architectural quant à lui, a été violemment critiqué, la nouvelle construction ayant entraîné la perte d'un chef-d'œuvre d'art musulman très apprécié par les érudits français et les spécialistes de l'architecture qui ont longtemps déploré cette perte.

Cette étude a voulu mettre l'accent sur les différentes phases du chantier de la construction de la cathédrale d'Alger, la conversion d'une mosquée en premier lieu et son adaptation aux besoins du culte catholique, son agrandissement suivant la nouvelle trame urbaine, sa démolition, et enfin sa reconstruction selon le modèle des églises du $19^{\mathrm{e}}$ siècle. On comprend le processus par lequel s'est fondée la première cathédrale, son contexte historique et urbain, à l'image de la ville d'Alger, qui elle aussi, subissait parallèlement une métamorphose totale, particulièrement dans centre de la ville. Au final, la superposition des sources donne l'opportunité d'avoir une lecture assez complète du palimpseste Ketchawa qui fait toute la particularité de ce monument emblématique.

Amel BeLlaLa

École polytechnique d'architecture et d'urbanisme d'Alger Enseignant-chercheur, architecte du patrimoine

31. Henri Klein, Algérie catholique, op. cit., p. 10-12.

32. Nadia Oulebsi, op. cit., p. 252.

33. AAA/283, op. cit.

34. Vigouroux et Ph. Caillat, Alger. Projet d'une nouvelle ville dressé et présenté le 20 janvier 1858 à Son Excellence monsieur le maréchal Randon, Alger, 1858, dans Nadia Oulebsir, op. cit., p. 133. 
